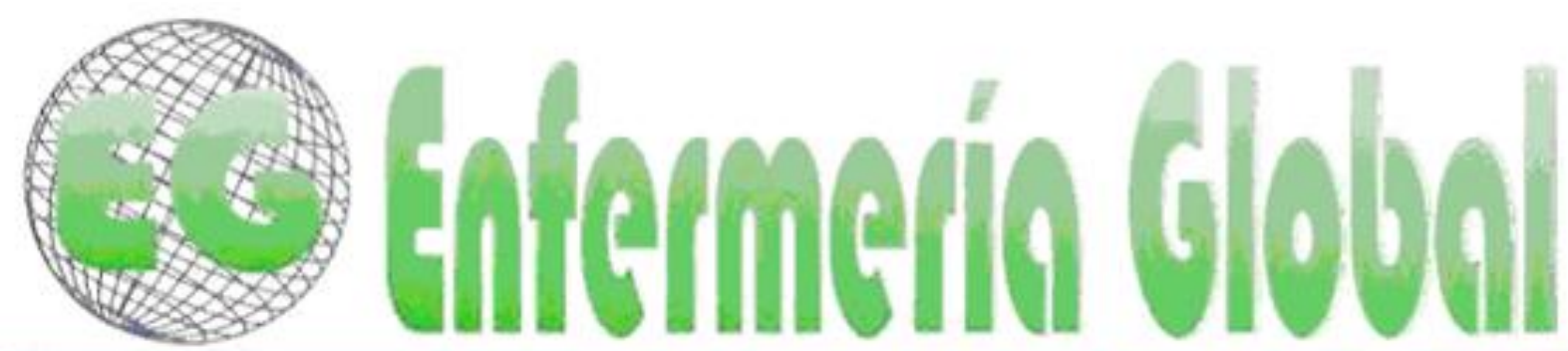

\title{
CLÍNIGA
}

\section{Prevalencia de obesidad infantil y hábitos alimentarios en educación primaria}

Prevalence of childhood obesity \& eating habbits in primary education

\author{
*Alba-Martín, Raquel \\ *Enfermera. Especialista em Salud Mental. Experto universitario en Investigación enfermera. USM \\ Hospital Universitario Reina Sofía, Córdoba. E-mail: raquelalbamartin@satse.es
}

Palabras clave: Obesidad Infantil; Hábitos alimentarios; Familia; Nutrición; Educación para la salud Keywords: Child Obesity; Eating Habits ; Family; Nutrition; Health Education.

\section{RESUMEN}

Introducción: En los países desarrollados la obesidad infantil se ha incrementado alarmantemente en los últimos años, convirtiéndose en un grave problema de salud pública. La obesidad es una enfermedad crónica de origen multifactorial que comienza durante la niñez y adolescencia. Se produce por un desequilibrio entre el consumo y el gasto energético. Como causas influyentes destacan: factores genéticos, biológicos, de comportamiento y culturales. Su tratamiento resulta muy complicado en esta etapa de crecimiento porque cualquier déficit nutricional puede repercutir negativamente en el desarrollo integral del niño.

Objetivos: Clasificar a la población de estudio en función de su Indice de Masa Corporal (IMC), establecer la relación que existe entre obesidad infantil y el uso del servicio de comedor escolar y verificar que el menú del comedor escolar es adecuado en cuanto al contenido calórico y el porcentaje de principios inmediatos.

Metodología: Se realizó un estudio observacional descriptivo transversal sobre obesidad en niños de 6 años. Para la recogida de datos, se utilizó una báscula para medir el peso y una cinta métrica para tallarlos, y con los datos de peso y estatura recogidos se calculó el IMC. Por otro lado, para poder comprobar el menú, el personal del comedor escolar nos facilitó un menú mensual.

Resultados: Dentro de los porcentajes europeos, nuestra muestra reflejó niños con sobrepeso y obesidad y también un considerable porcentaje de niños con bajo peso.

Conclusiones: Destacar el papel fundamental de la familia y cuidadores en los comedores escolares; fomentando hábitos saludables (ejercicio y dieta equilibrada) para poder prevenir patologías en la edad adulta secundarias a la obesidad, lo cual es una gran estrategia de seguridad para la salud pública y para la economía de los Sistemas Sanitarios. 


\section{ABSTRACT}

Introduction: In developed countries child obesity alarmingly in recent years, becoming in a serious problem of public health. Obesity is a chronic illness with multifactorial origin that begining in childhood and adolescence, produced it by an inbalance between intake and energy expenditure. Its causes could be genetic,biological, behavioral or cultural factors. Treatment is difficult in this growth stage because any nutritional deficit can negatively impact in children's comprehensive development.

Objectives: ClassifiedppilsBody Mass Index (BMI), observed relations between infant obesity and using school dining-room and checked that menu was appropriate in calories content and percentage of macronutrients.

Methodology: It has implemented an observational, descriptive and cross study about obesity in children six years. For data collection, it used a scale to measure weight and a tape measure to carve, and weight data collected height and BMI was calculated. Furthermore, in order to check the menu, the staff cafeteria gave us a monthly menu.

Results: Into europe percentages, our sample showed children with overweight and obesity and a considerable percentage with underweight.

Conclusions: Family and carers play significant rol in school dining-room promoting healthy habits (with exercise and a balance diet) to prevent pathologies in adulthood secondaries to obesity, which is an security strategy in public health and for economy of health systems.

\section{INTRODUCCIÓN}

La obesidad es considerada como la enfermedad del siglo XXI por las dimensiones adquiridas a lo largo de las últimas décadas, y por el impacto sobre la morbimortalidad, la calidad de vida y el gasto sanitario. Según la OMS, se define obesidad cuando el Índice de Masa Corporal (IMC) es igual o superior a 30, resultante de dividir el peso en kilogramos entre la estatura en metros al cuadrado. ${ }^{1-4}$

También se considera signo de obesidad un perímetro abdominal aumentado en hombres mayor o igual a $102 \mathrm{cms}$ y en mujeres mayor o igual a $88 \mathrm{cms}$.

Otra forma de cuantificar la obesidad, es mediante el porcentaje de grasa que tiene la persona. ${ }^{5}$ El peso graso total es la suma de peso de grasa más el peso residual. En las chicas, el peso graso oscila entre $18-20 \%$ y por encima de $30 \%$ ya sería peligroso y en los chicos oscila en $12-18 \%$ y por encima del $23 \%$ es ya un riesgo.

La obesidad es una enfermedad crónica de origen multifactorial que suele comenzar durante la niñez y la adolescencia. Esta enfermedad se caracteriza por acumulación excesiva de grasa o hipertrofia general del tejido adiposo en el cuerpo. Las causas de la obesidad son complicadas e incluyen factores genéticos, biológicos, del comportamiento y culturales. La obesidad puede ser de causa endógena (genética) o exógena (hábitos de vida). Básicamente, la obesidad ocurre por un desequilibrio entre el consumo y el gasto de energía.

La obesidad en los niños y adolescentes puede estar relacionada con diversos factores, entre ellos citamos los siguientes: binging ${ }^{6}$ (perder la capacidad para parar de comer), falta de ejercicio, historial de obesidad en la familia, enfermedades médicas (problemas endocrinológicos o neurológicos), medicamentos (esteroides y algunos medicamentos psiquiátricos), cambios en la vida que les causan mucho estrés (separaciones, divorcio, mudanzas, fallecimientos de familiares), problemas familiares, baja autoestima, depresión u otros problemas emocionales. Dentro de la 
clasificación etiológica de la obesidad se encuentra la asociada a los síndromes dismórficos $^{4}$, con alteraciones genéticas como el de Bardet-Biedl, Laurence-Moon, Prader Will, entre otros. La causa más frecuente es la exógena, debida a una ingesta de energía mayor de la necesaria, con una dieta no equilibrada asociada a disminución de la actividad física.

La obesidad forma parte del síndrome metabólico siendo un factor de riesgo para varias enfermedades: cardiovasculares, diabetes mellitus tipo 2, apnea del sueño, ictus, osteartritis, así como algunas formas de cáncer, sobre todo las hormonodependientes, ya que hay un buen almacenamiento graso para sintetizar estrógenos.

En niños entre 5-14 años es difícil la medición de sobrepeso y obesidad porque no hay una definición estandarizada y clara de obesidad infantil. Los niños que son obesos entre los seis meses y siete años de vida tienen probabilidad de seguir siendo obesos porque las células que almacenan la grasa (adipocitos) se multiplican en esta etapa.

En la infancia, la acumulación excesiva de grasa en el cuerpo implica dificultad para respirar, interferencias en el sueño, problemas ortopédicos, trastornos cutáneos, transpiración excesiva, hinchazón de pies y tobillo, mayor riesgo de enfermedad coronaria, diabetes, asma, cáncer, entre otros. Además figuran los problemas psicológicos provocados tanto por la discriminación social como por los problemas de autoestima.

El interés por la obesidad infantil se ha acrecentado en los últimos años en todos los países desarrollados. Suscita gran inquietud el que su prevalencia haya aumentado a lo largo de los últimos 20 años entre 2 y 3 veces. En la población infantil española se estima su prevalencia en alrededor de un 13\%. 7. Hoy en día, sabemos que entre un 16 y un $33 \%$ de los niños y adolescentes europeos son obesos. Los niños con sobrepeso tienden a convertirse en adultos con sobrepeso, a menos que adopten y mantengan unos patrones más saludables.

Generalmente un niño no se considera obeso hasta que pesa por lo menos un $10 \%$ más del peso recomendado para su estatura y complexión.

El incremento de la obesidad es debido a nuevos hábitos y estilos de vida adoptados secundarios a la incorporación de la mujer al mundo laboral, la celeridad del ritmo de vida, el incremento de familias desestructuradas, como consecuencia de esto se ha pasado a consumir platos precocinados de alto valor calórico o realización de dietas desequilibradas, por no tener tiempo suficiente para cocinar; pasando así de la dieta mediterránea a la comida rápida. También consecuencia de todo esto es un aumento del sedentarismo infantil, ya que se prioriza la utilización del videojuego, la conexión a Internet, ver la televisión, durante un número excesivo de horas al día, descuidando así la práctica de ejercicio diario.

Es por este motivo, que nos planteamos llevar a cabo un estudio sobre obesidad infantil.

Nuestros objetivos fueron:

1. General: 
- Clasificar a la población de estudio en función de su IMC.

\section{Específicos:}

- Determinar la relación que existe entre la prevalencia de la obesidad infantil y el uso del servicio de comedor escolar.

- Verificar que el menú del comedor escolar es adecuado en cuanto al contenido calórico y los porcentajes de glúcidos, lípidos y proteínas.

\section{METODOLOGÍA}

El estudio se realizó en un Colegio Público de Córdoba, a niños de educación primaria, para tratar de cuantificar el porcentaje de niños obesos y ver la similitud con los porcentajes europeos.

En este centro no existían experiencias previas respecto a este tipo de estudio, aunque a nivel nacional e internacional son numerosos los estudios que hay sobre la obesidad infantil y relacionando con los nuevos hábitos de vida que se les inculca a la generación de las nuevas tecnologías.

\section{Diseño}

Realizamos un estudio observacional, descriptivo, transversal sobre el IMC en niños de 6 años. Se desarrolló un tipo de muestreo intencional y no aleatorio definido de antemano a partir de los objetivos del estudio. Como criterio de inclusión se consideró únicamente que trajesen el consentimiento informado firmado por los padres. No se consideraron criterios de exclusión.

Para la recogida de datos, se utilizó una báscula para medir el peso y una cinta métrica para tallarlos, y con los datos de peso y estatura recogidos se calculó el IMC. Por otro lado, para poder comprobar el menú, el personal del comedor escolar nos facilitó un menú mensual.

Para que los niños pudiesen participar en el estudio, los padres debían rellenar previamente el correspondiente consentimiento informado, en el que se le garantiza la protección de los datos y el anonimato. Al tratarse de menores no se consintió que aceptaran la participación verbalmente.

La recogida de datos se llevó cabo durante los meses de enero-febrero de 2013 y fueron analizados mediante el programa estadístico SPSS versión 18.0, calculándose las frecuencias absolutas, relativas y porcentajes para las variables cualitativas. (Anexo 1)

Se les informó del proyecto y de los criterios de selección de la muestra, para que ellos pudieran informar a los cuidadores y les invitasen a participar. Además debían entregarles un documento informativo y el consentimiento informado 8 y solicitarles un teléfono de contacto al que más tarde llamarían los investigadores, salvo que los cuidadores expresasen su negativa, para aportar más información si fuese necesario y solicitar su colaboración, concretando la fecha y el lugar de la entrevista de manera flexible y adaptada a las circunstancias de los participantes. Se pretendía que la captación fuera lo menos complicada posible, para facilitar la participación de los cuidadores y no favorecer la pérdida de potenciales participantes. 


\section{RESULTADOS}

Tabla 1: Resultados según IMC en porcentaje.

\begin{tabular}{l|ccc}
\hline & \multicolumn{3}{c}{ Porcentajes obtenidos } \\
\hline IMC & OBESOS & DELGADEZ & SOBREPESO \\
Prevalencia & $10,5 \%$ & $11 \%$ & $15 \%$ \\
\hline
\end{tabular}

Fuente: Datos de la investigación. Elaboración propia.

En el estudio, finalmente, participaron 75 alumnos, de los cuales, el $38.67 \%$ eran niños y el $61.33 \%$ niñas. En la muestra, se obtuvo que un $10,5 \%$ de los participantes presentaba obesidad. De estos, el $6 \%$ corresponde con el sexo femenino y el $4,5 \%$ con el masculino.

Por otro lado, el $15 \%$ de los participantes presentó sobrepeso, siendo el $8 \%$ del sexo femenino y el $7 \%$ del sexo masculino. Otro porcentaje, el $11 \%$, tenían bajo peso o peso insuficiente, lo que indicaba delgadez extrema ( $7,5 \%$ niñas y $3,5 \%$ niños). De la totalidad de participantes, tan sólo una el $12 \%$ hacía uso del comedor escolar. Estos presentaban normo-peso.

Al comprobar el menú mensual, se verificó que la dieta era adecuada en cuanto a la cantidad calórica y a la proporción de macronutrientes (glúcidos, lípidos, proteínas: $55 \%, 30 \%$ y $15 \%$ respectivamente.

Figura 2: Resultados obtenidos estratificados por género.

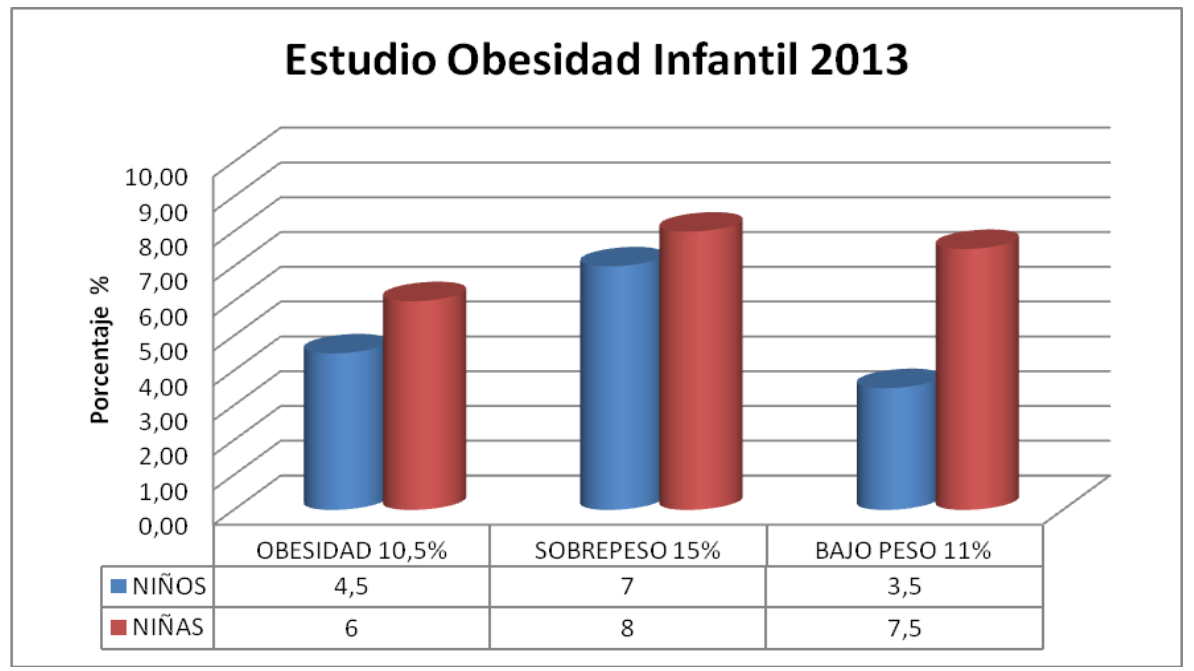


Figura 3: Resultados de la investigación. Elaboración propia.
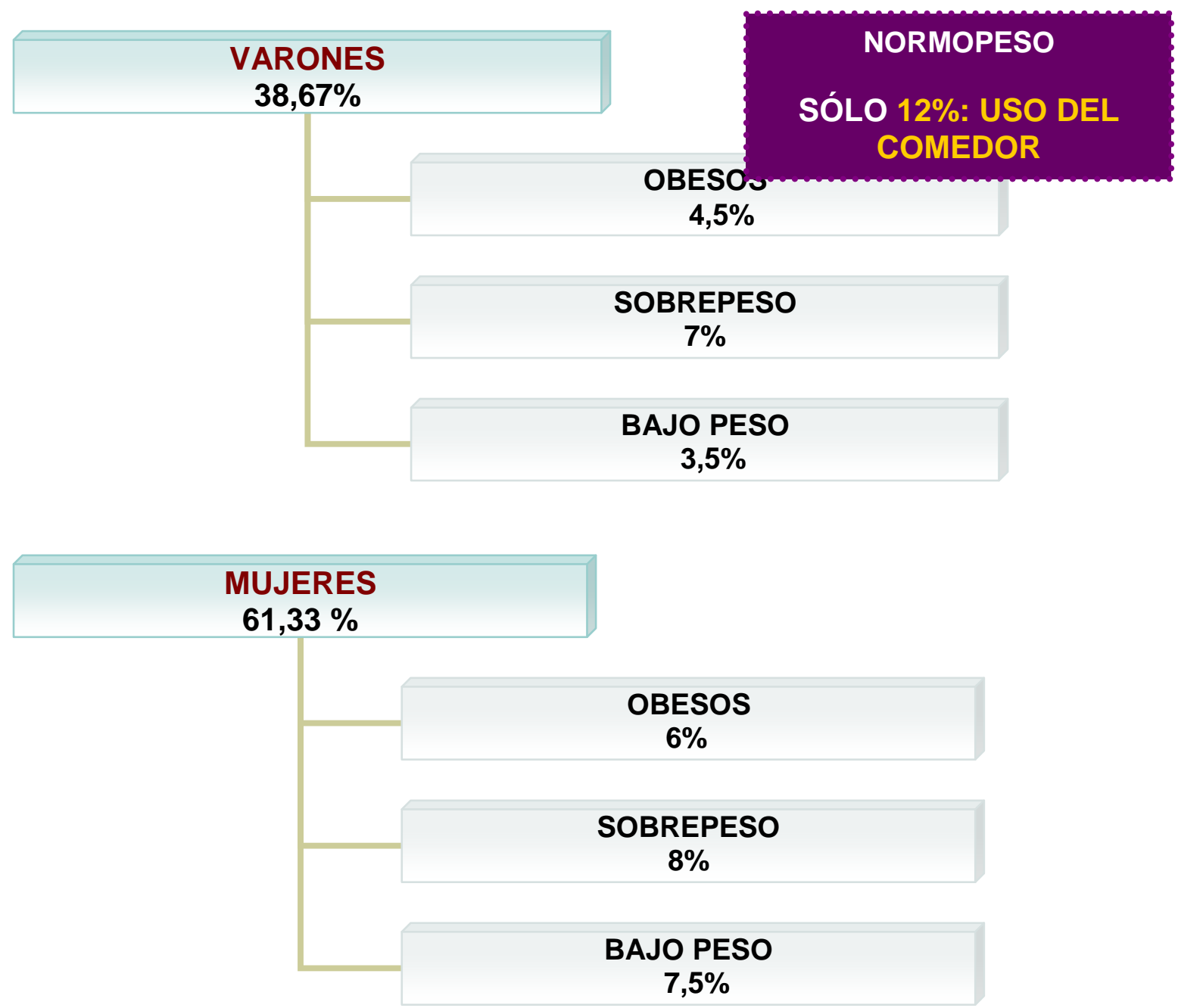

\section{DISCUSIÓN}

Analizando los datos obtenidos, podemos afirmar que el conjunto de niños obesos y niños con sobrepeso suman un tercio del total de la muestra de estudio, que es un valor a considerar. Respecto a los niños con delgadez extrema $(\mathrm{IMC}<18)$, al ser también un porcentaje elevado, sería interesante someterlos a un estudio para ver si su estado nutricional es saludable.

Debemos destacar que los escolares que usan el comedor están en normo-peso, cabe pensar que es por el cumplimiento de la dieta, implantada en menús, que se lleva a cabo en el centro. Es importante que desde la escuela se eduque en salud y se desarrolle este eje transversal creando unos hábitos saludables para el bienestar físico, psíquico y social de los niños y con ello prevenir la obesidad durante la infancia y sus posibles complicaciones en el futuro. ${ }^{9-13}$

Así, un análisis de la publicidad alimentaria dirigida a menores en 2008 encontró que el $71,2 \%$ de las comunicaciones comerciales de alimentos y bebidas emitidas durante la franja horaria de protección reforzada anunciaban productos de perfil nutricional pobre ("menos saludables"), según el modelo de perfil nutricional de Reino Unido ${ }^{3-11 .}$ El consumo de alimentos azucarados (postres) y bebidas tiene una correlación 
positiva con la incidencia de sobrepeso y obesidad en preescolares y niños mayores $^{10}$.

El nuevo código de corregulación (PAOS, 2012) firmado por la Agencia Española de Seguridad Alimentaria y Nutrición, contempla por vez primera la publicidad por Internet y pretende dar respuesta al artículo 46 de la Ley de Seguridad Alimentaria y Nutrición 1. Asimismo, dispone "el establecimiento de códigos de conducta que regulen las comunicaciones comerciales de alimentos y bebidas dirigidas a la población menor de quince años, con el fin de contribuir a prevenir la obesidad y promover hábitos saludables". Sin embargo, la restricción de su aplicación a los menores de 12 años, en medios audiovisuales e impresos, contraviene lo estipulado en la ley, pues permite, por ejemplo, que la publicidad alimentaria por televisión dirigida a los mayores de 12 años pueda hacer uso de personajes conocidos para el público en general que gocen de un alto grado de popularidad entre el público infantil.

Resulta cada vez más evidente que las tácticas de las industrias alimentarias y de la publicidad para oponerse con fuerza a la regulación estatal y promover la autorregulación por vía de acuerdos voluntarios son similares a las utilizadas previamente por las industrias del tabaco y el alcohol, cuya autorregulación se ha demostrado inefectiva y contraproducente para la salud pública. No debería sorprendernos, puesto que los intereses económicos de ambas industrias están directamente ligados al incremento del consumo de los productos anunciados, en su mayoría altamente energéticos y pobres en nutrientes, en clara contraposición con los objetivos de salud pública.

Por tanto, si se desea contribuir eficazmente a prevenir la obesidad infantil y promover hábitos saludables, urge establecer mecanismos de regulación estatal que prohíban cualquier tipo de publicidad dirigida a menores de alimentos y bebidas ricos en grasas saturadas, ácidos grasos trans, azúcares libres o sal.

Por otro lado, el tratamiento de esta enfermedad en esta etapa de la vida resulta muy complicado por varios motivos:

1. Se trata de una etapa de crecimiento con intensos cambios madurativos físicos y psicológicos en la que cualquier déficit nutricional puede repercutir negativamente.

2. La falta de autonomía en estas edades depende de la colaboración de la familia y del medio escolar.

\section{Barreras detectadas durante el desarrollo}

Debido a condiciones económicas actuales, ha disminuido el número de niños que utilizan el servicio del comedor escolar. Preocupa, igualmente, toda una amplia serie de patologías que, concomitantemente con la obesidad, se van instalando en la infancia. Tales son la diabetes tipo 2, diversos problemas respiratorios, el síndrome metabólico, la esteatosis hepática, o el pseudotumor cerebri, entre otros. Aceptando con seguridad que, en su origen, las circunstancias que producen el desarrollo de la obesidad infantil son de naturaleza exógena, las posibilidades de una acción preventiva han de ir dirigidas a modificar aquellas, en el sentido de mejorar las formas y modos de la alimentación infantil, así como sus comportamientos sociales y lúdicos ${ }^{7}$ 


\section{Oportunidad de participación del paciente y familia}

Aconsejamos la participación activa por parte de la familia porque juega un papel fundamental en las diferentes etapas de crecimiento / desarrollo del niño. Además es importante considerar que a partir de esta edad es cuando se instauran los principales hábitos como son la dieta y el ejercicio, por lo que se debe considerar un buen momento para incidir en ellos.

El hecho de prevenir la obesidad es importante desde el punto de vista de la seguridad de la salud pública y de la economía del sistema sanitario, puesto que se pueden prevenir numerosas patologías en la edad adulta. ${ }^{9}$

También consideramos oportuna en esta lucha la participación del sistema educativo para prevenir la obesidad infantil, deberían unificar los criterios junto con las instituciones sanitarias para fomentar hábitos de vida saludables, fomentando la participación y modelo adecuado familiar ambas partes. ${ }^{11}$

\section{Propuestas de líneas de investigación}

Repetir este estudio en un par de años y valorar la incidencia especialmente de la utilización del comedor escolar y el porcentaje de niños con obesidad y delgadez extrema, respecto a la evolución de la situación económica. ${ }^{12}$

\section{CONCLUSIONES}

La obesidad es un problema de salud y de estética que suele estar asociado a discriminación en la sociedad actual, ocasionándole al niño obeso problemas físicos y psicológicos, presentando baja autoestima, dificultad para las habilidades sociales, entre otros. Creemos importante que el personal docente detecte dicha discriminación y tome medidas al respecto. Nuestro porcentaje de niños obesos está por debajo de la media europea, pero si consideramos a los niños con sobrepeso como niños con riesgo de desarrollar obesidad, sí se encontraría nuestro estudio dentro del porcentaje europeo.

\section{REFERENCIAS}

1- Ley 17/2011 de Seguridad Alimentaria y Nutrición. 2011. (Consultado: 20/12/2014).

2- Organización Mundial de la Salud. Conjunto de recomendaciones sobre la promoción de los alimentos y bebidas no alcohólicas dirigida a los niños. Ginebra: Organización Mundial de la Salud; 2010:14.

3- Vásquez F, Díaz E, Lera L. Estudio longitudinal de la composición corporal por diferentes métodos como producto de una intervención integral para tratar la obesidad en escolares chilenos. Nutrición. Scielo- España. 2013.

4- Rodríguez G, Gallego S, et al. Uso del índice de masa corporal para valorar la obesidad en niños y adolescentes. Revista Española de Obesidad. 2006; 4: 284-8.

5- Chrzanowska M, Koziel S, Ulijaszek SJ. Changes in BMI and the prevalence of overweight and obesity in children and adolescents in Cracow and Poland. Economics and Human Biology. 2007.

6- Pichot P. Manual Diagnóstico y Estadístico de los Trastornos Mentales DSM IV-TR. 4ª Edición. Editorial Masson. 2009. 
7- Casado de Frías E. Obesidad en la infancia: Preocupaciones actuales. Anales de la Real Academia de Medicina. Madrid. TOMO CXXIII (Cuaderno Segundo). 2006: 34966.

8- Ley 41/2002, básica reguladora de la Autonomía del paciente de 14 de noviembre): Consentimiento informado.2002.

9- Saavedra JM, Dattilo AM. Factores alimentarios y dietéticos asociados a la obesidad infantil: recomendaciones para su prevención antes de los dos años de vida. Rev Peru Med Exp Salud Pública. 2012; 29(3):379-85.

10- Dubois L, Farmer AP, Girard M, Peterson K. Preschool children's eating behaviours are related to dietary adequacy and body weight. Eur J Clin Nutr. 2007; 61(7):846-55.

11-Romero T. ¿Pueden contribuir las industrias alimentaria y de la publicidad a prevenir la obesidad infantil y promover hábitos saludables? Rev. Gaceta Sanit. Barcelona. 2013; 27: (6).

12-Morales P, Santos JL, González A. Validación factorial de un cuestionario para medir la conducta de comer en ausencia de hambre y su asociación con obesidad infantil. Revista chilena Scielo. Chile. 2012.

13-Romero T. Un nuevo método para detectar en niños y adolescentes el riesgo de complicaciones por obesidad. ICM. Veganomicón. 2014. 
ANEXO 1: Cronograma. Recogida de datos.

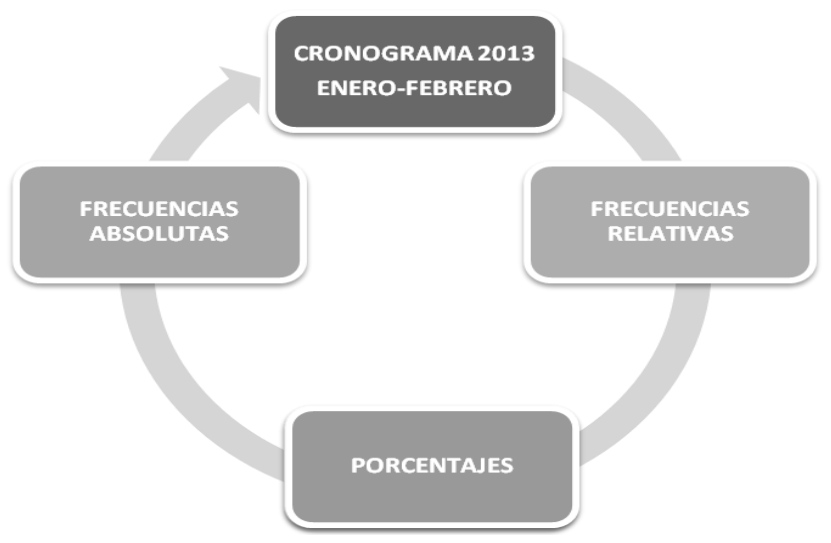

ANEXO 2: Índice de QUETELET.

IMC Ó BMI (Body Mass Index) : PESO (Kilogramos) /(TALLA*(Metros))2

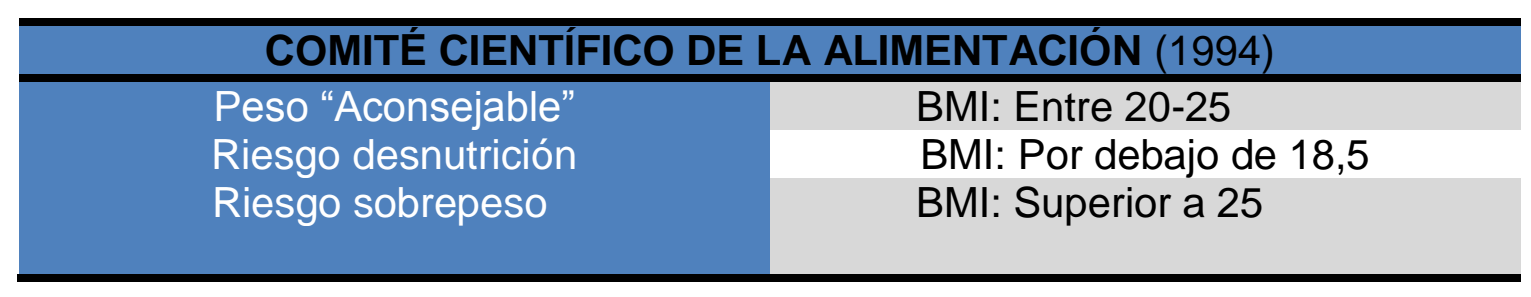

Fuente: Elaboración propia.

ANEXO 3: Patrones alterados en la obesidad como TCA.

\begin{tabular}{cc}
\hline TRASTORNOS ALIMENTARIOS (TCA) & OBESIDAD \\
\hline NIVEL DE PESO & $20 \%$ de sobrepeso \\
ATRACONN & Ocasional \\
CONTROL DE PESO & Dietas restrictivas \\
DISTORSIÓN IMAGEN CORPORAL & No \\
ANSIEDAD POST-COMIDAS & No \\
RELACIÓN ÁNIMO-ATRACONES & No \\
PSICOPATOLOGÍA SECUNDARIA & Normal a moderada \\
\hline
\end{tabular}

Fuente: Tomada de Belloch y cols. (2008) 
Yo, padre/madre de

Con DNI

He tenido oportunidad de efectuar preguntas sobre el estudio en cuestión. He recibido respuestas satisfactorias y suficiente información.

He hablado con los encargados. Con dirección de contacto: colegio_fm@hotmail.com También he sido informado de forma clara, precisa y suficiente de los siguientes extremos que afectan a los datos personales que se contienen en este consentimiento y en la ficha o expediente que se abra para la investigación:

$\square \square$ Estos datos serán tratados y custodiados con respeto a la intimidad de mi hijo y a la vigente normativa de protección de datos.

$\square \square$ Sobre estos datos me asisten los derechos de acceso, rectificación, cancelación y oposición que podré ejercitar mediante solicitud ante el investigador responsable en la dirección de contacto que figura en este documento.

$\square$ Estos datos no podrán ser cedidos sin mi consentimiento expreso.

Doy mi consentimiento sólo para la extracción necesaria en la investigación de la que se me ha informado, exclusivamente en ella, sin posibilidad de compartir o ceder éstas, en todo o en parte, a ningún otro investigador, grupo o centro distinto del responsable de esta investigación o para cualquier otro fin. Comprendo que puedo retirarlo del estudio: Cuando quiera, sin tener que dar explicaciones, sin que esto repercuta en los cuidados médicos de mi hijo. Declaro que he leído y conozco el contenido del presente documento, comprendo los compromisos que asumo y los acepto expresamente. $\mathrm{Y}$ por ello, firmo este consentimiento informado de forma voluntaria para:

MANIFESTAR MI DESEO DE DEJARLE PARTICIPAR en el estudio sobre obesidad infantil en el Colegio público de Córdoba. Al firmar este consentimiento no renuncio a ninguno de mis derechos.

FDO:

${ }^{1}$ DOCUMENTO DE C.I. FUENTE: ELABORACIÓN PROPIA. 
Recibido: 15 de noviembre 2014; Aceptado: 16 de enero 2015 